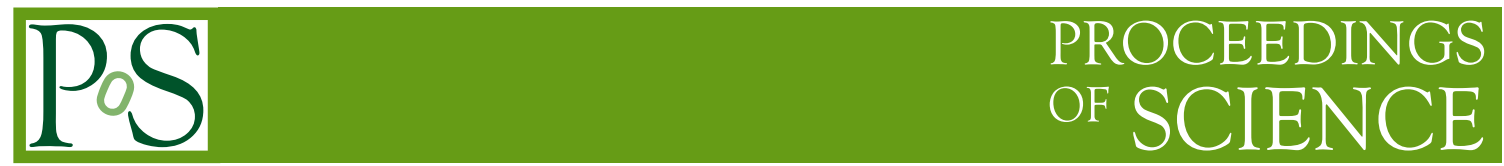

\title{
Non-thermal hard X-ray emission from M87
}

\section{R. Walter*}

INTEGRAL Science Data Centre, Observatoire de Genève, Université de Genève, Chemin d'Ecogia 16, CH-1290 Versoix, Switzerland

E-mail: Roland.Walter@unige.ch

\section{A. Neronov}

INTEGRAL Science Data Centre, Observatoire de Genève, Université de Genève, Chemin

d'Ecogia 16, CH-1290 Versoix, Switzerland

We report the detection of the radio galaxy M87 in the 20-60 keV energy band by INTEGRAL. The detected flux cannot be explained by the high-energy tail of the thermal X-ray emission from the core of Virgo cluster. The signal can also not be explained by non-thermal emission from the nucleus and kpc-scale jet of M87 in quiescence. We argue that plausible explanations of the observed flux could be the HST-1 knot when flaring or the extended non-thermal emission from the large-scale radio halo of M87. These two alternate explanations can be tested with future monitoring of the source with INTEGRAL.

7th INTEGRAL Workshop

September 8-11 2008

Copenhagen, Denmark

${ }^{*}$ Speaker. 


\section{Introduction}

The giant elliptical galaxy M87 hosts a variety of thermal and non-thermal phenomena. It is the $\mathrm{cD}$ galaxy at the center of the Virgo cluster and the bulk of the observed X-rays (1-10 keV) is the thermal emission from the cluster core (1). M87 is also one of the best studied radio galaxies, its $2 \mathrm{kpc}$ long core radio jet was the first extragalactic jet discovered and its variable X-ray emission is known to be of synchrotron origin $(2 ; 3)$.

The nucleus and the jet are surrounded by a larger scale (tens of kpc) expanding radio halo of complicated morphology (4). The halo is interacting with the thermal X-ray emitting plasma at the core of the Virgo cluster, presumably heating the intracluster gas.

Hard X-ray (above $20 \mathrm{keV}$ ) emission can be produced by one or several components of M87 (the nucleus, the jet, the halo, the intracluster gas). However, until now, M87 was never detected at hard X-rays as instruments did not had enough spatial resolution to separate it cleary from the closeby bright Seyfert 2 galaxy NGC 4388 (see Fig 1) (5) or lacked sensitivity. We report a detection of M87 at hard X-rays achieved thanks to the IBIS/ISGRI imager (6; 7) on board INTEGRAL (8). Its imaging capability (angular resolution of 12') and sensitivity turn out to be sufficient to separate the signal of the two sources.

Combining these data with observations obtained with EPIC $(9 ; 10)$ on board XMM-Newton (11) at lower energies allows to constrain the nature of the observed hard X-ray emission. The only plausible sites of the observed hard X-ray emission are the HST-1 radio knot and the large, $10 \mathrm{kpc}$ scale, radio halo of M87. The high-energy electrons which produce the radio synchrotron emission in the halo are also emitting in the $20-60 \mathrm{keV}$ band via inverse Compton scattering of the dense stellar radiation field in the $\mathrm{cD}$ galaxy.

\section{Hard X-ray observations of M87}

\subsection{INTEGRAL observations and data analysis}

Several INTEGRAL core-programme and open-time observations have covered the sky region centered on M87. We selected all available pointings located within 15 degrees of M87, which resulted in an ISGRI effective good exposure (corrected for dead-time and off-axis absorption) of $300 \mathrm{ks}$. Sky images were created in the band $20-60 \mathrm{keV}$. Analysis has been performed with the Offline Science Analysis software, version 6.0, using standard parameters. Good time intervals were built using a strong constraint on the attitude stability (deviation $<2$ arcsec). Image cleaning was performed fixing source positions and was applied independently of the source strength, allowing for negative source model. To build the best possible mosaic of the field we excluded the outskirts of individual images as well as pointing images which had background fluctuations larger than 1.1 in significance. The properties of the mosaic image are presented in (12).

M87 is detected in the $20-60 \mathrm{keV}$ mosaic image (Fig. 1 Left) with an average count rate of $0.19 \pm 0.04 \mathrm{cts}^{-1}$ two orders of magnitude below that of NGC 4388. The source significance is $5.1 \sigma$ for a flux of $F_{20-60 \mathrm{keV}}=(8.6 \pm 1.8) \times 10^{-12} \mathrm{erg} \mathrm{cm}^{-2} \mathrm{~s}^{-1}$ (assuming $\left.\Gamma=2.15\right)$. The ISGRI image of M87 can be fitted with a gaussian of width $\sigma=7.3 \pm 3.2 \operatorname{arcmin}(90 \%$ confidence). This is consistent with the width of the ISGRI point spread function, the image does not differ from that of a point source. 

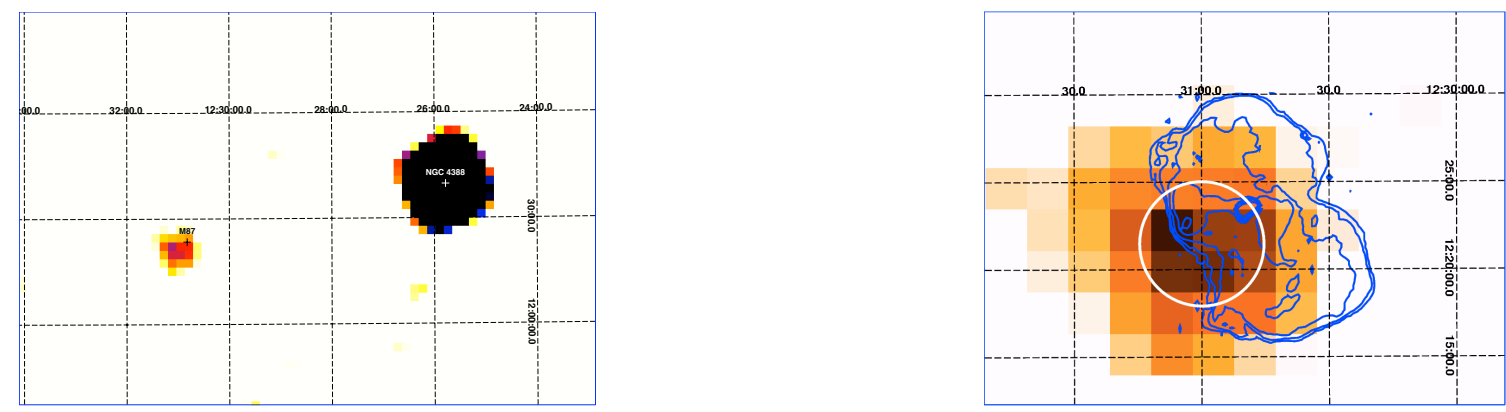

Figure 1: Left: $20-60 \mathrm{keV}$ significance image of the region around M87. The bright source 1.3 degree away from M87 is the Seyfert 2 galaxy NGC 4388. The catalogue source positions are indicated. M87 is detected with a significance of 5.1 sigma. Right: The contour plot of the large scale radio halo around M87 (from 4) overlayed on the INTEGRAL image of M87. The white circle indicate the INTEGRAL error circle.

The best fit position of the hard X-ray image of M87 (fixing the width of the gaussian to that of the point spread function) $\alpha=12: 31.0 \delta=12: 21$ (J2000; 90\% statistical uncertainty: 3.6 arcmin) is marginally consistent with the position of the core of M87. The data indicate a possible displacement of the hard X-ray emission towards the South-East when compared to position of the core radio jet. We divided the data in three time periods to search for source variability. The source significance is too low for the data to indicate any sign of variability.

\subsection{Comparison with previous hard X-ray observations}

The Virgo cluster and M87 have been observed by several hard X-ray missions. The hard X-ray emissions from M87 and NGC 4388 could be somewhat separated during scanning observations performed with the Ginga Large Area Counter experiment. While the soft X-ray emission appeared extended and centered on M87, the hard X-ray emission appeared displaced towards NGC 4388. (13) deconvolved the signal, measured the contribution of both galaxies and derived a flux of $F_{20-60 \mathrm{keV}}=(4 \pm 1) \times 10^{-11} \mathrm{erg} \mathrm{cm}^{-2} \mathrm{~s}^{-1}$ for M87, assuming $\Gamma=1.75$. Contrasting with the above, the hard X-ray observations performed with the Proportional Counter Array of RXTE failed to detect a non thermal component in M87 with an upper limit of $F_{20-60 \mathrm{keV}}<0.3 \times 10^{-11} \mathrm{erg} \mathrm{cm}^{-2} \mathrm{~s}^{-1}$ (assuming $\Gamma=2 ; 14$ ).

These inconsistent values, derived from collimator instruments, depend on the accurate modelling of the geometrical response, of the cosmic X-ray background and of other sources in the field. (14), concluded that these instruments did not convincingly detected any hard X-ray tail in M87. Using the University of Birmingham coded mask X-ray telescope on board spacelab 2, (5) obtained the first high resolution hard X-ray images of the field which allowed them to derive an upper limit of $F_{20-60 \mathrm{keV}}<1.0 \times 10^{-11} \mathrm{erg} \mathrm{cm}^{-2} \mathrm{~s}^{-1}$ for M87 (assuming $\Gamma=1.75$ ). The INTEGRAL/ISGRI detection of M87 we are reporting here is consistent with this, however the upper limit derived from RXTE appears too low, unless the hard X-ray flux of M87 is variable.

\section{Origin of the hard X-ray emission}

There are several potential sites of hard X-ray emission in M87: the intracluser medium in the core of Virgo cluster, the nucleus of M87, the kpc-scale core radio jet and the $10 \mathrm{kpc}$-scale 
radio halo. The relatively low effective exposure time (300 ks) available on M87 with INTEGRAL is not sufficient to distinguish between these different possibilities using geometrical arguments alone. However, as we will show in the subsequent sub-sections, a comparison of the 1-10 keV spectral properties of the components spatially resolved in the soft X-ray band enables to identify the probable site of the $20-60 \mathrm{keV}$ emission.

\subsection{Thermal emission from the core of Virgo cluster}

The X-ray emission from M87 in the soft X-rays is dominated by the thermal emission from the core of the Virgo cluster. The Virgo cluster is known to be one of the prototypical examples of the "cooling core" clusters, in which a complicated interaction between the hot gas, cooling due to the X-ray emission and the active nucleus of the cluster cD galaxy, which apparently heats the gas, is observed (1). In spite of the fact that the soft X-ray luminosity of the cluster core is very large the low temperature of the X-ray emitting gas, which varies between 0.8 and $2.7 \mathrm{keV}$ in the inner $30^{\prime}$ region, excludes to explain the observed $20-60 \mathrm{keV}$ flux as a high-energy tail of the thermal spectrum.

In order to verify this, we have extracted the spectra from concentric rings $0^{\prime}-3^{\prime}, 3^{\prime}-6^{\prime}, 6^{\prime}-$ $9^{\prime}, 9^{\prime}-12^{\prime}$ and $12^{\prime}-15^{\prime}$ around the center of M87 from the XMM-Newton observation. We have fitted the spectra of the outer rings $\left(6^{\prime}-15^{\prime}\right)$ with a 1-temperature thermal emission model (vmekal) and the spectra of the inner rings $\left(0^{\prime}-6^{\prime}\right)$ with a 2-temperature model to take into account that a cooler component is observed in the core of the Virgo cluster (15). We have calculated the thermal flux in the $20-60 \mathrm{keV}$ energy band, predicted by the model fits in each of the concentric rings and compared the predicted level of thermal emission to the level of the flux detected by INTEGRAL. The result is shown in the left pannel of Fig. 2, The predicted thermal contribution to the INTE$G R A L$ flux does not exceed several percent, even if one collects the flux from a $30^{\prime}$-diameter region around the center of M87. The extrapolation of the non thermal component detected by INTEGRAL in the X-ray band is compatible with the spectra observed by XMM-Newton.
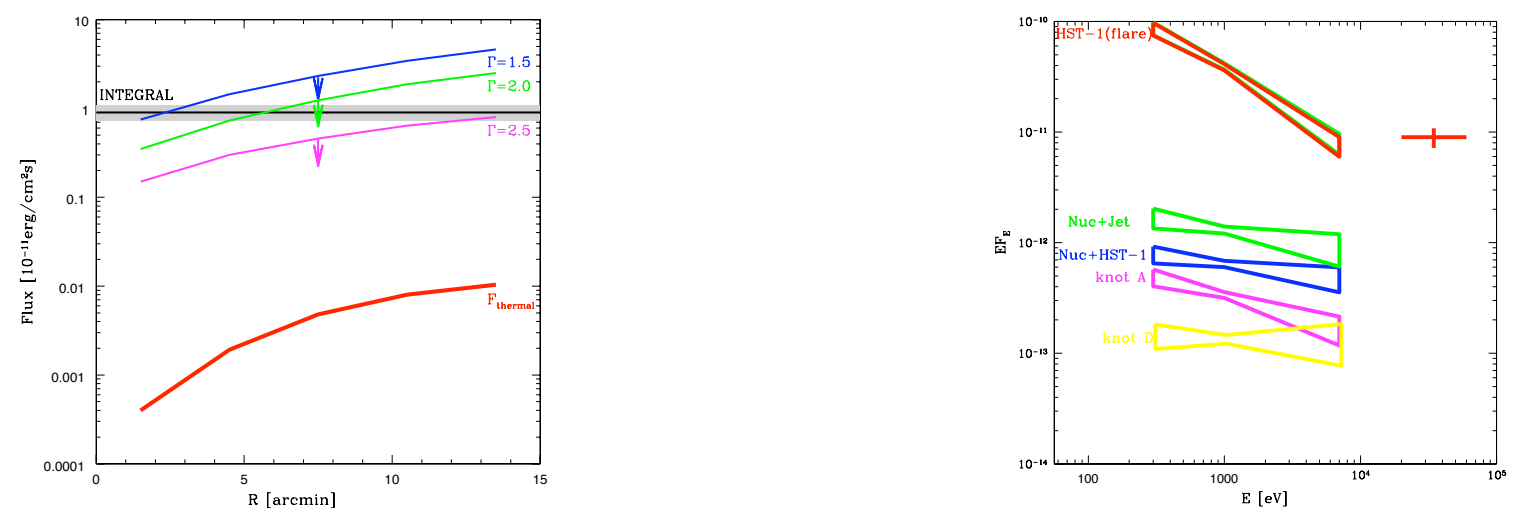

Figure 2: Left: Comparison of the INTEGRAL flux in 20-60 keV energy band with the predictions for the thermal (from the intracluster medium of Virgo cluster) and powerlaw (from the radio halo) contributions to the flux, based on the analysis of XMM-Newton data. The curves show the overall flux expected from the region $0<r<R$. The upper limits for the powerlaw contributions are shown for three different values of the photon index $\Gamma$. Right: Spectra of the nucleus and bright knots of the 2-kpc scale jet, measured by Chandra, as compared to the flux level detected by INTEGRAL. 


\subsection{The nucleus and the kpc-scale jet}

The 1-10 keV spectrum of the unresolved X-ray source in the nucleus of M87 has a flux density of $8.0 \pm 0.2 \times 10^{-13} \mathrm{erg} \mathrm{s}^{-1} \mathrm{keV}^{-1}(1 \mathrm{keV})$ and exhibits a soft spectrum with a photon index $\Gamma=2.23 \pm 0.04$ (16). Extrapolation of this spectrum to higher energies would result in the prediction of the 20-60 keV flux $(3.6-5.6) \times 10^{-13} \mathrm{erg} \mathrm{cm}^{-2} \mathrm{~s}^{-1}$, which is more than an order of magnitude lower than the observed flux (see Fig. 2 right). Thus, unless the spectrum of the nuclear source exhibits a sharp turn-over at the energy $10 \mathrm{keV}$, the possibility that the observed hard X-ray emission is produced by the nuclear source is ruled out.

The possibility that the emission is produced by one of the knots of the kpc-scale jet of M87 (except for the knot HST-1, see below) is ruled out by a similar argument: unless the spectrum of the knots has a sharp hardening at $10 \mathrm{keV}$, the extrapolation of the observed soft X-ray flux into the 20-60 keV band results in an order-of-magnitude under-prediction of the hard X-ray flux. The same is true for the collective emission from the nucleus and the set of knots of the jet (see Fig. 2 right).

The possibility that the hard X-ray emission can be produced in the knot HST-1 of the M87 jet requires a special analysis. This knot has exhibited a strong long-duration flare during the period of operation of INTEGRAL. During the flare, which lasted for about a year, the emission from HST-1 has dominated the X-ray flux from the M87 jet. The spectrum of the synchrotron emission emitted by HST- 1 from the radio to the optical energy band can be well approximated by a powerlaw with a spectral index $\alpha_{r-o} \simeq 0.45$. The overall flux of the knot has exhibited a variation by a factor of $\sim 30$, from $\sim 3 \times 10^{-13} \mathrm{erg} \mathrm{cm}^{-2} \mathrm{~s}^{-1}$ up to $\sim 9 \times 10^{-12} \mathrm{erg} \mathrm{cm}^{-2} \mathrm{~s}^{-1}$ during the period $2000-2005$ (see Fig. 3). The X-ray spectrum of the HST-1 knot also varied in the course of the flare. A hardening of the spectrum with the spectral index changing from $\alpha_{X} \simeq 1.35 \pm 0.1$ to $\alpha_{X}=1.05 \pm 0.07$ was observed in May 2002. However, because of the duration of the flare, a detailled monitoring of the $\mathrm{X}$-ray spectral evolution during the flare is lacking. In principle, an extrapolation of the spectrum of the knot to the $20-60 \mathrm{keV}$ band, assuming $\alpha_{X} \simeq 1-1.5$ gives a prediction for the $20-60 \mathrm{keV}$ flux, which is compatible with the level of the flux, detected by INTEGRAL (see Fig. 3 left).

An identification of the source of the emission in 20-60 keV band with the knot HST-1 looks plausible. An obvious prediction of such scenario is that the flux in the $20-60 \mathrm{keV}$ band should be strongly variable. Taking into account the marginal significance of the INTEGRAL detection, one can only make a prediciton that the source should only be detectable in 2005, at the peak of activity of HST-1. However, the analysis of the actual source behaviour in the $20-60 \mathrm{keV}$ band shows that this may not be the case.

\subsection{Diffuse emission from the radio halo of M87}

An other possible counterpart for the hard X-ray source is the emission from the large (10-kpc) scale non-thermal radio halo (larger scale jet and radio lobes) around M87 (4). The angular extent of the halo is $\geq 10$ arcmin. The contours delimiting the boundaries of the large scale radio $(90 \mathrm{~cm})$ halo are overlayed over the INTEGRAL image in Fig. 3 right. One can see that the position of the hard X-ray source is compatible with the position and extent of the radio halo.

In order to constrain the size and/or spectral characteristics of the hard X-ray emission from the radio halo we have added a powerlaw component to the thermal emission components, while fitting 

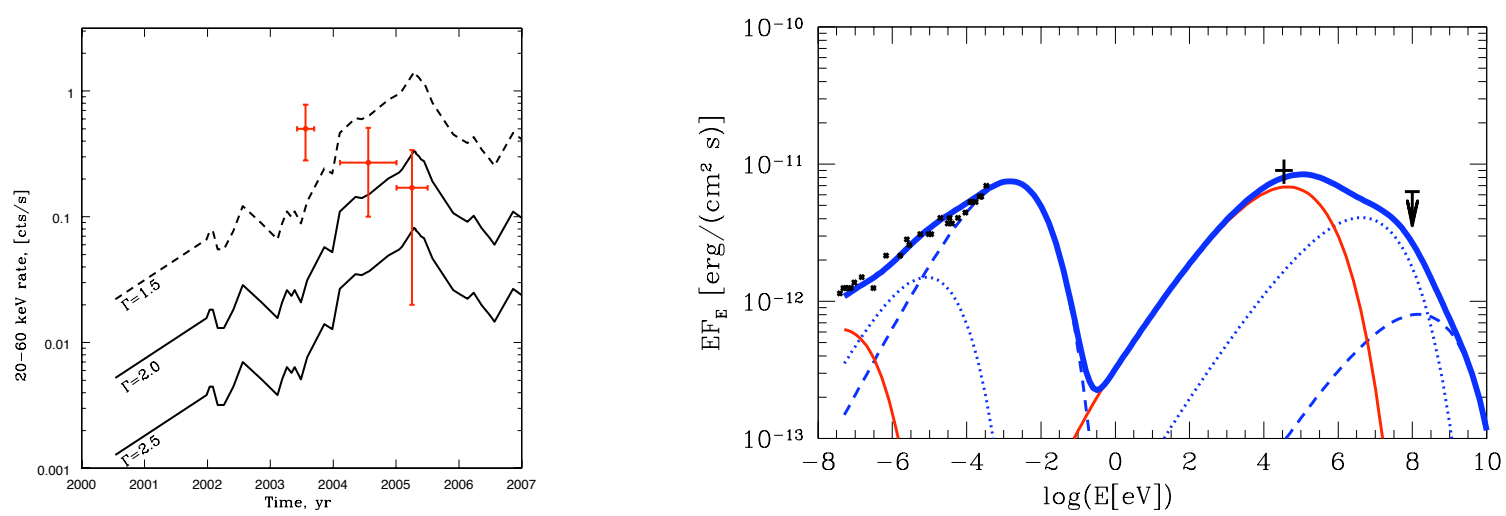

Figure 3: Left: The lightcurve of M87 in the 20-60 keV energy band compared to the lightcurve of the knot HST-1 of the M87 jet observed by Chandra. Right: The inverse Compton model for the hard X-ray emission from the radio halo of M87. The radio data are from NED. The EGRET upper limit on the signal from M87 is shown by an arrow. Red thin solid line shows the synchrotron and inverse Compton emission from electrons with the spectrum $d N / d E \sim E^{-2} \exp \left(-E / E_{\text {cut }}\right)$ with $E_{\text {cut }}=1 \mathrm{GeV}$ in the magnetic field $B=3 \mu \mathrm{G}$, in the outer part of the halo. Blue, dotted line shows the spectrum of electrons with the same spectrum, but with the cut-off at $E_{0}=10 \mathrm{GeV}$ and in magnetic field $B=7 \mu \mathrm{G}$. Blue, dashed line shows the spectrum of emission for $E_{0}=0.1 \mathrm{TeV}$ and $B=10 \mu \mathrm{G}$, from the inner part of the halo. Thick, solid, blue line shows the overall spectrum of the halo.

the 0.5-10 keV XMM-Newton spectra extracted from the concentric rings around the center of M87. Since the emission in this energy band is completely dominated by the thermal emission, it is not possible to constrain the properties of the powerlaw component. Instead, are have estimated the upper limits on the powerlaw contributions to the spectra, assuming a certain value of the photon index $\Gamma$. The results are shown in Fig. 2 right. One can see that under the assumption of a hard powerlaw, $\Gamma=1.5$, the flux detected by INTEGRAL can come from the innermost region $0<r<3^{\prime}$ of the radio halo. In the case of $\Gamma=2$, the size of the emission region is $0<r<5^{\prime}$, which is about the size of the radio halo. At the same time, in the case of the soft powerlaw, $\Gamma=2.5$, the size of the emission region, $R \sim 13^{\prime}$ turns out to be larger than the size of the radio halo. This provides an indication that the $20-60 \mathrm{keV}$ spectrum of the halo is rather hard, $\Gamma<2.5$.

In the galactic bulge of M87, the energy density of the soft radiation field is dominated by the infrared $\left(\varepsilon_{0}=0.1-1 \mathrm{eV}\right)$ photons emitted by stars, with a typical radial profile $U_{\text {bulge }}=$ $L_{\text {bulge }} /\left(4 \pi R_{\text {bulge }}^{2}\right) \approx\left(100 \mathrm{kpc} / R_{\text {bulge }}\right) \mathrm{eV} / \mathrm{cm}^{3}$ between 1 and $10 \mathrm{kpc}(17)$. Within a $10 \mathrm{kpc}$ scale region, occupied by the radio halo, the stellar photon density is more than 40 times larger than the density of the $\mathrm{CMB}$ photons, $U_{\mathrm{CMB}} \simeq 0.25 \mathrm{eV} / \mathrm{cm}^{3}$.

If inverse Compton scattering takes place, the energy of the electrons emitting at an energy $\varepsilon$ is $E_{e}$, IC $\simeq 300\left[0.1 \mathrm{eV} / \varepsilon_{0}\right]^{1 / 2}[\varepsilon / 20 \mathrm{keV}]^{1 / 2} \mathrm{MeV}$. If the magnetic field in the halo is of the order of $B \sim 10 \mu \mathrm{G}$, the synchrotron radiation from these electrons is emitted at a frequency $\simeq 100\left[E_{e, \mathrm{IC}} / 300 \mathrm{MeV}\right]^{2}[B / 10 \mu \mathrm{G}] \mathrm{MHz}$.

The ratio of the inverse Compton (hard X-ray) and synchrotron (radio) emission of the source can be estimated from the ratio of the radiation and magnetic fields energy densities $L_{\mathrm{IC}} / L_{\mathrm{S}}=$ $8 \pi U_{\mathrm{rad}} / B^{2} \simeq 4\left[U_{\mathrm{rad}} / 10 \mathrm{eV} / \mathrm{cm}^{3}\right][B / 10 \mu \mathrm{G}]^{-2}$. To compare this to the observed hard X-ray and radio luminosities of the entire halo, one needs to integrate the surface brightness at the frequen- 
cies $v \sim 100 \mathrm{MHz}$ over the image of the large scale halo. Although not always cited in the original papers, such integrated flux is available from the M87 spectrum published in the NASA Extragalactic Database (NED). In Fig. 3 right, we reproduce the overall radio spectrum of M87 as inferred from the NED. One can see that the radio flux at $100 \mathrm{MHz}$ is at the level of $10^{-12} \mathrm{erg} / \mathrm{cm}^{2} \mathrm{~s}$. As the detected $\mathrm{X}$-ray flux is an order of magnitude higher, one finds an average magnetic field strength $B \leq 6\left[R_{\text {bulge }} / 10 \mathrm{kpc}\right]^{-1} \mu \mathrm{G}$ in the inverse Compton emission region. This constraint is consistent with the estimates of the magnetic field in the halo from the radio data, $B \sim 6-10 \mu \mathrm{G}$ (obtained from the minimal energy requirement, 4).

In principle, the density of the infrared/optical photon background, the magnetic field strength and the electron $100 \mathrm{MeV}-1 \mathrm{GeV}$ spectrum could vary significantly across the $10 \mathrm{kpc}$ scale halo. Thus, the regions of strongest radio synchrotron emission do not necessarily coincide with the regions of the strongest inverse Compton emission in the hard X-ray band. In fact stronger inverse Compton emission is expected to come from the regions of lower magnetic fields in the halo.

Both the radio and X-ray images of the extended emission in M87 reveal the existence of two 10-kpc-scale "arms", to the East and to the South-West directions $(4 ; 18)$. The direction and extent of these arms are shown in the right pannel of Fig. 1. The arms were interpreted by (18) as being due to a directed motion (or oscillations around a mean position) of M87 through the intracluster gas of the core of Virgo cluster. The suggested direction of motion is towards the the North-West. It is interesting to note that, although the precision of the source localization with INTEGRAL is not sufficient to firmly establish a displacement of the centroid of the extended hard X-ray source from the center of M87, one can note that the INTEGRAL source is, apparently brighter on the South-East side of the halo, on the opposite side to the suggested direction of the relative motion of the nucleus of M87 with respect to the Virgo cluster.

The typical dynamical scale for the motion of the intracluster medium in the central $D \sim$ $10-100 \mathrm{kpc}$ region of the Virgo cluster can be estimated as $D / v_{\mathrm{vir}} \sim 0.01-0.1 \mathrm{Gyr}$, where $v_{\mathrm{vir}} \sim$ $10^{8} \mathrm{~cm} / \mathrm{s}$ is the typical virial velocity in the central part of the cluster. This time scale turns out to be comparable to the inverse Compton cooling time, $t_{\mathrm{IC}} \simeq 0.1\left[10 \mathrm{eV} / \mathrm{cm}^{3} / U_{\mathrm{rad}}\right]\left[300 \mathrm{MeV} / E_{e}\right] \mathrm{Gyr}$. This means that if the nucleus of M87 moves through the intracluster medium, the inverse Compton emission from particles ejected from the nucleus is expected to reveal a "trace" of the trajectory of the nucleus. In this respect, in the inverse Compton scenario, a displacement of the position of the centroid of the hard X-ray source from the position of the nucleus of M87 is expected. To verify if this is indeed the case, a more accurate localisation of the hard X-ray source is required, which is possible with more INTEGRAL exposure on the source.

Since the relative importance of inverse Compton and synchrotron emission from the 0.1$1 \mathrm{GeV}$ electrons is expected to vary across the halo, only qualitative picture of the broad-band spectrum can be established. A detailed calculation could be done only if a numerical model of the dynamics of matter and magnetic fields in the outflow from the nucleus of M87, moving through the intracluster medium, is available. A qualitative model of the broad band spectrum of the halo is shown in Fig. 3 right. The spectrum of the hard X-ray emission region is shown by a red thin solid line. The spectrum of electrons is assumed to have the form $d E \sim E^{-2} \exp \left(-E / E_{\text {cut }}\right)$ with $E_{\text {cut }}=1 \mathrm{GeV}$. The magnetic field is $B=3 \mu \mathrm{G}$. One can expect that in the bright radio features in the halo (such as the features A-H in Fig. 3 of 4 ) the magnetic field is enhanced, which leads to a higher synchrotron flux and lower inverse Compton emission from these regions. Closer to 
the nucleus/jet of M87, the electrons should have higher cut-off energies and, as a result, their synchrotron contribution is increased and extended to higher energies, while the strength of the inverse Compton contribution decreases. This is illustrated by the dotted and dashed blue lines in Fig. 3 right (the cut-off in the electron spectrum used for the calculation of the spectrum shown by the dashed line is $E_{0}=10 \mathrm{GeV}$ ). The overall spectrum of the halo is the sum of the contributions of different regions, such as the one shown by the thick blue solid line in Fig. 3.

\section{Conclusions}

We have shown that the non thermal hard X-ray emission from M87 detected by INTEGRAL could be related to the HST-1 knot or to the non-thermal emission from the large, 10-kpc scale radio halo around its active nucleus. This non-thermal emission is not visible in the $1-10 \mathrm{keV}$ energy band, where the flux is strongly dominated by the thermal emission from the intracluster medium of the Virgo cluster. Above $20 \mathrm{keV}$ the expected thermal contribution (the high-energy tail of the thermal bremsstrahlung spectrum with the temperature $T \sim 2 \mathrm{keV}$ ) to the flux is more than an order of magnitude below the detected flux level (see Fig. 2).

The 20-60 keV emission can be produced by inverse Compton scattering of infrared photons in the galactic bulge of M87, by the same population of electrons which emit synchrotron in the radio band (see Fig. 1). In this scenario, the magnetic field in the hard X-ray emission region (of the size of ten(s) of kiloparsecs around the nucleus of M87) is constrained to be not stronger than $\sim 6 \mu \mathrm{G}$, which is consistent with the estimates, obtained from the radio data. The life-time of the 0.1-1 GeV electrons, responsible for the inverse Compton emission in the hard X-ray band is comparable to the dinamical scale of motions of the intracluster medium in the core of Virgo cluster. This means that if the nucleus of M87 is moving relatively to the dynamical center of Virgo cluster, the hard X-ray emission can "trace" its trajectory over ten(s) of kiloparsecs. Fig. 1 shows that the hard X-ray source is possibly displaced from the center of M87 in the direction opposite to the direction of relative motion, previously suggested based on the analysis of the assymetry of the X-ray emission from the core of Virgo cluster by (18). This hypothesis can be tested with a better precision of localisation of the extended hard X-ray source which will become available with more INTEGRAL exposure on the source.

\section{References}

[1] Böhringer, H. et al., A\&A 365 (2001) L181.

[2] Marshall, H. L. et al., ApJ 564 (2002) 683.

[3] Harris, D. E. et al., ApJ 586 (2003) L41.

[4] Owen, F., Eilek, J., and Kassim, N., ApJ 543 (2000) 611.

[5] Hanson, C. G., Skinner, G. K., Eyles, C. J., and Willmore, A. P., MNRAS 242 (1990) 262.

[6] Ubertini, P. et al., A\&A 411 (2003) L131.

[7] Lebrun, F. et al., A\&A 411 (2003) L141. 
[8] Winkler, C. et al., A\&A 411 (2003) L1.

[9] Turner, M. J. L. et al., A\&A 365 (2001) L27.

[10] Strüder, L. et al., A\&A 365 (2001) L18.

[11] Jansen, F. et al., A\&A 365 (2001) L1.

[12] Paltani, S. et al., A\&A 485 (2008) 707.

[13] Takano, S. and Koyama, K., PASJ 43 (1991) 1.

[14] Reynolds, C. S., Heinz, S., Fabian, A. C., and Begelman, M. C., ApJ 521 (1999) 99.

[15] Sakelliou, I. et al., A\&A 391 (2002) 903.

[16] Di Matteo, T., Allen, S., Fabian, A., and A.S., W., ApJ 582 (2003) 133.

[17] Stawarz, L., Sikora, M., Ostrowski, M., and Begelman, M. C., ApJ 608 (2004) 95.

[18] Simionescu, A., Böhringer, H., Brüggen, M., and Finoguenov, A., ApJ 465 (2001) 749. 